\title{
Recurrent Generative Networks for Multi-Resolution Satellite Data: An Application in Cropland Monitoring
}

\author{
Xiaowei Jia ${ }^{1}$, Mengdie Wang ${ }^{1}$, Ankush Khandelwal ${ }^{1}$, Anuj Karpatne ${ }^{2}$, Vipin Kumar ${ }^{1}$ \\ ${ }^{1}$ Department of Computer Science and Engineering, University of Minnesota \\ ${ }^{2}$ Department of Computer Science, Virginia Tech \\ ${ }^{1}\left\{\right.$ jiaxx221, wang5699, khand035, kumar001\}@umn.edu, ${ }^{2}$ karpatne@vt.edu
}

\begin{abstract}
Effective and timely monitoring of croplands is critical for managing food supply. While remote sensing data from earth-observing satellites can be used to monitor croplands over large regions, this task is challenging for small-scale croplands as they cannot be captured precisely using coarseresolution data. On the other hand, the remote sensing data in higher resolution are collected less frequently and contain missing or disturbed data. Hence, traditional sequential models cannot be directly applied on high-resolution data to extract temporal patterns, which are essential to identify crops. In this work, we propose a generative model to combine multi-scale remote sensing data to detect croplands at high resolution. During the learning process, we leverage the temporal patterns learned from coarse-resolution data to generate missing high-resolution data. Additionally, the proposed model can track classification confidence in real time and potentially lead to an early detection. The evaluation in an intensively cultivated region demonstrates the effectiveness of the proposed method in cropland detection.
\end{abstract}

\section{Introduction}

Given the global population growth, an automated cropland monitoring system can offer timely agricultural information which is essential for managing the growing needs of food supply and food security. For example, grain production per acre in United States has doubled from 1975 to 2015 to meet the demand from growing population. These productivity gains are attributable to improved crop varieties and increased planting area. Both of these factors can be captured by an effective monitoring system. Moreover, monitoring croplands has a lot of implications for their environmental sustainability, e.g., the energy consumption for irrigation and the resulting contaminants.

Effective monitoring requires the ability to identify crops over large spatial regions and over long time periods. In recent years, the increasing availability of remote sensing data and advancements in machine learning have created an unrealized potential for analyzing land covers over space and time. Since most land covers have distinct seasonal temporal patterns, sequential models such as Recurrent Neural Networks (RNN) have been widely used to capture these patterns in land cover detection [Jia et al., 2017a; Lyu et al., 2016].

While these sequential models have shown success in leveraging temporal knowledge in classification, they are mostly designed for coarse-resolution remote sensing data, e.g. MODIS (250m, daily). However, the spatial resolution of such data is quite low which makes them unsuitable for monitoring small-scale farms that are quite common in many parts of the world. High-resolution data such as Sentinel $(10 \mathrm{~m}$, every 10 days in 2016) and Landsat (30m, every 16 days) can be used to monitor small farms, but they are captured less frequently compared to coarse-resolution data. This creates a major issue, especially because remote sensing data is often missing or of poor quality due to clouds and aerosols. For example, Sentinel data are supposed to be available every 10 days ( $\sim 36$ dates in a year), but are available for much less than 25 dates for many locations around the world. If such low quality data is directly used for classification, they are likely to produce bad results. For coarse-resolution data such as from MODIS, a common way to handle this issue is to create composites that aggregate data from multiple dates by selecting the data with the least noise. For example, MODIS 8-day composites are used quite frequently [Guindin-Garcia et al., 2012]. Since high-resolution data are available much less frequently, creating composites such as the ones used for MODIS will result in very infrequent data, making it difficult to capture dynamics of the phenomenon at desired time scale.

In this work, we present a novel framework, Multi-scale Analysis of Remote Sensing data with Missing or poor quality data (MARSM), that combines remote sensing data of different spatial scales, i.e. coarse-resolution and highresolution, to jointly detect/classify croplands. In particular, we utilize MODIS dataset as a coarse-resolution dataset, and Sentinel dataset as a high-resolution dataset. We choose Sentinel over Landsat for its more frequent availability (every 10 days) and better spectrum coverage. We develop a generative sequential model based on variational recurrent neural networks (VRNN) [Chung et al., 2015] on multi-scale data. This model leverages the temporal patterns from MODIS data to guide the learning process for Sentinel data.

The crop growing process often shows much variability since the crops in different places can have different grow- 
ing patterns (caused by weather conditions, amount of fertilizer, slope of the land, farmer behaviors). When handling such data with high variability, deterministic sequential models, such as the standard RNN, are known to suffer from the blurry prediction issue [Habibie et al., 2017; Mathieu et al., 2015] by averaging all the possible growing trajectories. In contrast, VRNN ia able to model the variability in crop growing trajectories by introducing latent variables in the internal probabilistic transition structure. For example, consider two corn locations $\mathrm{A}$ and $\mathrm{B}$ where the corn grows faster in A due to the applied fertilizers. The standard deterministic model will learn a growing pattern of corn to be the average of $\mathrm{A}$ and $\mathrm{B}$. In contrast, the VRNN model can automatically sample different latent variables to reflect the difference between $\mathrm{A}$ and $\mathrm{B}$ and then precisely capture the pattern of each location individually.

Next, we apply the generative process to produce missing Sentinel data. The generation of missing data enables the modeling of a complete crop growing process, which consequently contributes to a better classification. Given the variability in crop growing process, we enforce the generated Sentinel data to conform to true temporal transitions using the guidance of MODIS data and an adversarial regularizer.

Existing detection methods classify land covers only after collecting data from an entire year. However, it is of great interest to governments and companies to obtain the agricultural information at an early stage. We develop a progressive classification method using the outputs from the generative model to track classification confidence in real time. This method can help identify the most discriminative periods for each crop type and thus detect crops at an early stage.

To show the effectiveness in classifying crops and capturing the true growth patterns of each crop type, we evaluate the proposed framework in a crop-intensive region in southwestern Minnesota, US, where high-quality ground-truth is available from USDA Crop Data Layer ${ }^{1}$.

\section{Problem Definition}

In this work, we aim to classify each location at the resolution of a Sentinel pixel $(10 \mathrm{~m} \times 10 \mathrm{~m})$ into one of several major crop varieties. The input features include MODIS data (coarseresolution) and Sentinel data (high-resolution), both of which contain multiple time steps in a time period. We fix the time interval to be every 10 days (the same with Sentinel) and utilize MODIS composite images in each 10-day interval. More dataset details will be introduced in Section 4.

For each location, we represent its coarse-resolution MODIS input data as $X^{M}=\left\{x_{1}^{M}, x_{2}^{M}, \ldots, x_{T}^{M}\right\}$ with a total of $T$ time steps. For high-resolution Sentinel data, we assume each location has only one missing period between $t_{1}$ and $t_{2}$ in method discussion, i.e., $X^{S}=\left\{x_{1}^{S}, \ldots, x_{t_{1}}^{S}, x_{t_{2}}^{S}, \ldots, x_{T}^{S}\right\}$. However, our proposed method can be easily generalized to handle multiple missing periods in real-world datasets. In our implementation, $X^{M}$ and $X^{S}$ are the data from the MODIS pixel and the Sentinel pixel that cover this location.

Given the multi-scale sequential data collected from certain period, e.g. a year, our objective is to conduct a multi-

\footnotetext{
${ }^{1}$ https://nassgeodata.gmu.edu/CropScape/
}

class classification and output the posterior probability of class label, $p\left(y \mid X^{S}, X^{M}\right)$. Besides, we wish to model the progression of this posterior probability $p\left(y \mid x_{\leq t}^{S}, x_{\leq t}^{M}\right)$, also referred to as classification confidence, as time $t$ progresses. This enables the identification of crop types with reasonable accuracy before collecting all the data from the entire period.

\section{Method}

In this section, we propose a framework to combine multiscale sequential data for classifying crops. We first introduce a generative model for learning temporal patterns and handling missing data. Then we will describe a progressive classification method based on the generative model.

\subsection{Recurrent Generative Networks}

Since MODIS data and Sentinel data are captured using different optical sensors, we model them separately with two recurrent generative networks. However, we will later show the use of the temporal information learned from the MODIS sequence to assist the generative process of Sentinel data.

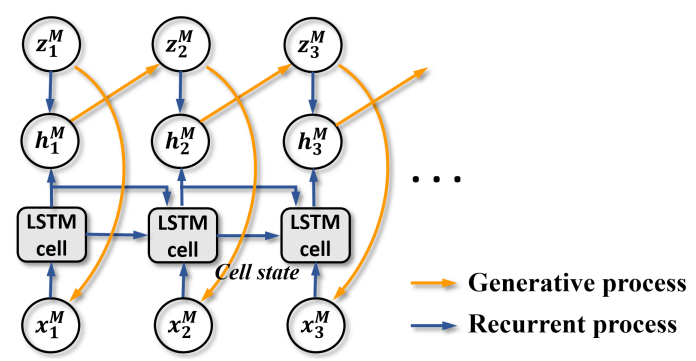

Figure 1: The generative modeling on MODIS sequential data.

We first build a variational recurrent neural networks (VRNN) on MODIS data (Fig. 1). We will now describe the generative process, recurrent process and inference in details.

The objective of the generative process is to estimate data likelihood $p\left(x^{M}\right)$. We will update model parameters through optimizing the variational lower-bound of log-likelihood. Then given any test data, we can conduct inference of the latent variables $z^{M}$, which are then used for classification.

\section{Generative Process}

At each time step $t$, VRNN retains the deterministic hidden representation $h_{t}^{M}$ in standard RNN to store the temporal information. While remote sensing data reflect crop growing process over time, they are also influenced by variability in environmental conditions (including weather as well as nutrients applied by farmers). Therefore, VRNN also introduces latent random variables $z_{t}^{M}$, which encode the knowledge of underlying crop type and the natural/human factors with influence on spectral features.

The generative process starts with sampling $z_{t}^{M}$ from a Gaussian distribution determined by the information at the previous time step $t-1$, as:

$$
\begin{aligned}
z_{t}^{M} & \sim \mathcal{N}\left(\mu_{0, t}^{M}, \operatorname{diag}\left(\left(\sigma_{0, t}^{M}\right)^{2}\right)\right), \\
{\left[\mu_{0, t}^{M}, \sigma_{0, t}^{M}\right] } & =\varphi^{\text {prior }}\left(h_{t-1}^{M} ; \tau^{\text {prior }}\right),
\end{aligned}
$$


where $h_{t-1}^{M}$ is the hidden representation at $t-1, \varphi^{\text {prior }}$ is a trainable function with parameter $\tau^{\text {prior }}$ (see Section 4.2), $\operatorname{diag}(\cdot) \operatorname{represents~a~diagonal~variance~matrix.~}$

Then we sample $x_{t}^{M}$, i.e., MODIS data features at $t$, from a Gaussian distribution with its mean and variance determined by $z_{t}^{M}$ through a function $\varphi^{g e n}$, as follows:

$$
\begin{aligned}
x_{t}^{M} \mid z_{t}^{M} & \sim \mathcal{N}\left(\mu_{x, t}^{M}, \operatorname{diag}\left(\left(\sigma_{x, t}^{M}\right)^{2}\right)\right), \\
{\left[\mu_{x, t}^{M}, \sigma_{x, t}^{M}\right] } & =\varphi^{g e n}\left(g\left(z_{t}^{M} ; \tau^{g}\right), h_{t-1}^{M} ; \tau^{g e n}\right) .
\end{aligned}
$$

\section{Recurrent Process}

The hidden representation $h_{t}^{M}$ at time $t$ is obtained through the recurrent procedure, as follows:

$$
h_{t}^{M}=\operatorname{Rec}\left(f\left(x_{t}^{M} ; \tau^{f}\right), g\left(z_{t}^{M} ; \tau^{g}\right), h_{t-1}^{M} ; \theta\right),
$$

where the function $\operatorname{Rec}(\cdot)$ is implemented using a LongShort Term Memory (LSTM) with parameter $\theta$ in our work, $f\left(x_{t}^{M} ; \tau^{f}\right)$ and $g\left(z_{t}^{M} ; \tau^{g}\right)$ extract features from raw MODIS data and latent variables (see Section 4.2 for more details).

Inference: The direct inference of $p\left(z_{t}^{M} \mid x_{t}^{M}\right)$ requires the marginalization over $z_{t}^{M}$, which is computationally intractable. Instead, VRNN approximates $p\left(z_{t}^{M} \mid x_{t}^{M}\right)$ by a Gaussian distribution $q\left(z_{t_{1}}^{M} \mid x_{t_{t}}^{M}\right)$ [Chung et al., 2015]. The mean and variance of $q\left(z_{t}^{M} \mid x_{t}^{M}\right)$ are determined by $x_{t}^{M}$ and $h_{t-1}^{M}$ through a function $\varphi^{i n f}$, as follows:

$$
\begin{aligned}
z_{t}^{M} \mid x_{t}^{M} & \sim \mathcal{N}\left(\mu_{z, t}^{M}, \operatorname{diag}\left(\left(\sigma_{z, t}^{M}\right)^{2}\right)\right), \\
{\left[\mu_{z, t}^{M}, \sigma_{z, t}^{M}\right] } & =\varphi^{i n f}\left(f\left(x_{t}^{M}\right), h_{t-1}^{M} ; \tau^{i n f}\right),
\end{aligned}
$$

\section{High-resolution Modeling}

Next, we utilize the temporal information obtained from MODIS data to guide the generative process of Sentinel data. Specifically, the latent variable $z_{t}^{S}$ for Sentinel data depends not only on the high-resolution information by $t-1$ (encoded by $h_{t-1}^{S}$ ), but also the temporal patterns of low-resolution data by time $t$ (encoded by $h_{t}^{M}$ ), as shown in Fig. 2 . The prior distribution of $z_{t}^{S}$ can be expressed as:

$$
\begin{aligned}
z_{t}^{S} & \sim \mathcal{N}\left(\mu_{0, t}^{S}, \operatorname{diag}\left(\left(\sigma_{0, t}^{S}\right)^{2}\right)\right), \\
{\left[\mu_{0, t}^{S}, \sigma_{0, t}^{S}\right] } & =\psi^{\text {prior }}\left(h_{t-1}^{S}, h_{t}^{M} ; \xi^{\text {prior }}\right),
\end{aligned}
$$

Then we sample $x_{t}^{S}$ from $z_{t}^{S}$ in a similar way with Eq. 2 . The generative process involves both the information from previous Sentinel data as well as the MODIS data until current time step. Similarly, the inference process should also involve $h_{t}^{M}$, which depends on $x_{\leq t}^{M}$. Therefore we have the approximated inference function as $q\left(z_{t}^{S} \mid x_{t}^{S}, x_{\leq t}^{M}\right)$.

\section{Variational Lower-bound}

the log-likelihood of multi-scale data can be factorized into two components, corresponding to coarse-resolution data and high-resolution data, respectively.

$\log p\left(x_{\leq T}^{M}, x_{\leq t_{1}, t_{2}: T}^{S}\right)=\log p\left(x_{\leq t_{1}, t_{2}: T}^{S} \mid x_{\leq T}^{M}\right)+\log p\left(x_{\leq T}^{M}\right)$,

where the subscript $\left\{\leq t_{1}, t_{2}: T\right\}$ represents all the available time steps for high-resolution data, i.e., the time steps before $t_{1}$ and from $t_{2}$ to $T$.

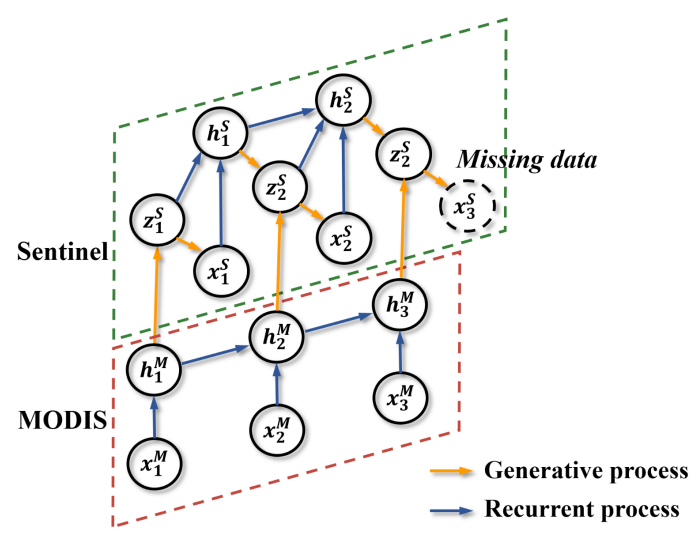

Figure 2: The generative process of Sentinel data and the use of the generative process in producing missing data.

Since the marginalization over $z$ is computationally intractable, we alternatively maximize the variational lowerbound of the log-likelihood. By using the fact that $q\left(z_{\leq T}^{M} \mid x_{\leq T}^{M}\right)=\prod_{t} q\left(z_{t}^{M} \mid x_{\leq t}^{M}, z_{<t}^{M}\right)$, and $p\left(x_{\leq T}^{M}, z_{\leq T}^{M}\right)=$ $\prod_{t} p\left(x_{t}^{M} \mid z_{<t}^{M}, x_{<t}^{M}\right) p\left(z_{t}^{M} \mid x_{<t}^{M}, z_{<t}^{M}\right)$, we can obtain a variational lower-bound for the coarse-resolution data:

$$
\begin{aligned}
& \log p\left(x_{\leq T}^{M}\right) \geq \\
& \sum_{t}\left\{-\mathbb{E}_{q\left(z_{<t}^{M} \mid x_{<t}^{M}\right)} \operatorname{KL}\left(q\left(z_{t}^{M} \mid x_{\leq t}^{M}, z_{<t}^{M}\right)|| p\left(z_{t}^{M} \mid x_{<t}^{M}, z_{<t}^{M}\right)\right)\right. \\
& \left.-\mathbb{E}_{q\left(z_{\leq t}^{M} \mid x_{\leq t}^{M}\right)} \log p\left(x_{t}^{M} \mid z_{\leq t}^{M}, x_{<t}^{M}\right)\right\} .
\end{aligned}
$$

Similarly, we can derive the variational lower-bound for the likelihood of high-resolution Sentinel data $\log p\left(x_{t=1: t_{1}, t_{2}: T}^{S} \mid x_{\leq T}^{M}\right)$ as follows:

$$
\begin{aligned}
& \log p\left(x_{\leq t_{1}, t_{2}: T}^{S} \mid x_{\leq T}^{M}\right) \\
& \geq \sum_{t \leq t_{1}, t_{2}: T}\left\{-\mathbb{E}_{q\left(z_{<t}^{S} \mid x_{<t}^{S, M}\right)} \operatorname{KL}\left(q\left(z_{t}^{S} \mid x_{\leq t}^{S}, x_{\leq t}^{M}, z_{<t}^{S}\right)|| p\left(z_{t}^{S} \mid x_{<t}^{S}, x_{\leq t}^{M}, z_{<t}^{S}\right)\right)\right. \\
& \left.-\mathbb{E}_{q\left(z_{\leq t}^{S} \mid x_{\leq t}^{S, M}\right)}^{S} \log p\left(x_{t}^{S} \mid z_{\leq t}^{S}, x_{<t}^{S}\right)\right\} .
\end{aligned}
$$

By approximating the expectation in Eqs. 7 and 8 by the "reparameterization trick" [Kingma and Welling, 2013], the variational lower-bound becomes fully differentiable and can be maximized by the standard back-propagation algorithm.

\subsection{Adversarial Data Generation}

It is noteworthy that the lower-bound in Eq. 8 only takes the summation over $t \leq t_{1}$ and $t_{2} \leq t \leq T$. However, to compute the distribution at time step $t_{2}$, we need the information of $x_{t_{1}+1: t_{2}-1}^{S}$ and $h_{t_{1}+1: t_{2}-1}^{S}$. Also, the information in missing period enables the modeling of a complete crop growing process and facilitates the progressive classification described in Section 3.3. Therefore, we generate missing data during the period $\left[t_{1}+1, t_{2}-1\right]$ in the following way. For each time step $t$ in $\left[t_{1}+1, t_{2}-1\right]$, we first generate $z_{t}^{S}$ by Eq. 5 using $h_{t-1}^{S}$ and $h_{t}^{M}$. Then we sample $x_{t}^{S}$ according to the Gaussian distribution determined by $z_{t}^{S}$ (Fig. 2). After obtaining $x_{t}^{S}$, we compute $h_{t}^{S}$ and the distributions at $t+1$. 
Due to the potential temporal changes caused by a variety of natural/human factors, a reasonable generative process may output sequential data following $t_{1}$ in various trajectories with different probabilities. To ensure that the generated data are consistent with the true scenario, we leverage two auxiliary information sources to constrain the data generation. First, as mentioned in Section 3.1, we utilize the coarseresolution data (MODIS) to guide the transition of Sentinel data, as MODIS data is more frequently available. Second, we impose an adversarial regularizer to enforce that the generated data do not deviate from the true data after $t_{2}$.

Specifically, we repeat the generative process to sample the data after $t_{2}$. The adversarial regularizer enforces that the generated data and the true data after $t_{2}$ cannot be easily distinguished by a well trained discriminative classifier $\mathcal{D}: z^{S} \mapsto[0$-true, 1-generated]. Our cost function for the generative model combines the log-likelihood (Eqs. 7 and 8) and the adversarial regularizer with a hyper-parameter $\lambda$, as:

$$
\begin{aligned}
\mathcal{J} & =-\log \left(p\left(X^{M}, X^{S}\right)\right)+\lambda \mathcal{L}_{\mathcal{D}}, \\
\mathcal{L}_{\mathcal{D}} & =\sup _{\mathcal{D}} \sum_{t \geq t_{2}} \mathbb{E}_{\hat{x}_{t}^{S} \mid x_{\leq t}^{S}{ }_{1}, x_{\leq t}^{M}} \log \mathcal{D}\left(z_{t}^{S} \mid \hat{x}_{t}^{S}\right)+\mathbb{E}_{\mathcal{X}_{t}^{S}} \log \left(1-\mathcal{D}\left(z_{t}^{S} \mid x_{t}^{S}\right)\right),
\end{aligned}
$$

where $\hat{x}$ denotes the generated data. $\mathcal{X}_{t}^{S}$ represents the distribution of provided Sentinel data at time $t$. The selection of hyper-parameters will be discussed in Section 4.2.

\subsection{Progressive Classification}

After gathering latent variables from the generative model, we utilize them to capture the progression of classification confidence over time. Specifically, we utilize the accumulated discriminative information until time $t$ to make classification decision at $t$. We expect that the model becomes more and more confident about the classification as time progresses. For location $i$, the discriminative information at time $t$ is computed from its obtained latent variables $\left[z_{i, t}^{S}, z_{i, t}^{M}\right]$. Here we add the subscript $i$ to represent the location index. To mitigate the noise at individual locations, we also adopt an isotropic Gaussian smoothing over the spatial neighborhood $\mathcal{N}(i)$. In the smoothing process, we use latent variables learned from MODIS data since MODIS data are more robust to noise. More formally, we collect the discriminative information at time $t$ and add it to an accumulated variable $o_{i, t}$, as:

$o_{i, t}=o_{i, t-1}+\sum_{j \in \mathcal{N}(i)} \frac{\exp \left\{-\left\|z_{i, t}^{M}-z_{j, t}^{M}\right\|^{2} / 2 \gamma^{2}\right\}}{\left(2 \pi \gamma^{2}\right)^{d_{m} / 2}} \operatorname{sigm}\left(W\left[z_{j, t}^{S}, z_{j, t}^{M}\right]\right)$,

where $\gamma^{2}$ represents the isotropic variance in the smoothing process, and $d_{m}$ is the dimensionality of $z^{M}$. W denotes the parameters in sigmoid function to transform latent variables $\left[z_{j, t}^{S}, z_{j, t}^{M}\right]$ to the discriminative information at $t$.

Then we compute the posterior probability of class label $y_{i}$ by time step $t$, via a softmax function, as:

$$
p\left(y_{i} \mid x_{\leq t}^{S}, x_{\leq t}^{M}\right)=\operatorname{softmax}\left(o_{i, t}\right)
$$

The entire framework can be trained using backpropagation algorithm in two stages. In the first stage, we conduct unsupervised training by minimizing the cost function in Eq. 9. Then in the second stage, we fine-tune model parameters in supervised fashion with training labels.

\section{Experiment}

\subsection{Datasets}

MODIS. We combine MODIS MOD09A1 and MOD09Q1 multi-spectral products, collected by MODIS instruments onboard NASA's satellites. MODIS data are collected for every single day. The dataset provides reflectance values on 7 spectral bands $(620-2155 \mathrm{~nm})$ for every location at $250 \mathrm{~m}$ resolution. The product MOD09A1 and MOD09Q1 prerprocess the satellite data by filtering precipitable water and cloud. To match the temporal frequency of Sentinel data, we utilize MODIS composite images by selecting per-pixel reflectance values with least noise during each time interval.

Sentinel. Sentinel-2A data are collected by European Space Agency, which aims to provide global data for every 10 days. The Sentinel-2A data product performs a cloud screening, but does not conduct atmospheric corrections. The data consist of reflectance values on 13 spectral bands, including the visible spectrum, NIR and SWIR. Depending on the spectral bands, the spatial resolution of collected data is $10 / 20 / 60 \mathrm{~m}$. In our work, we project all the bands into $10 \mathrm{~m}$ spatial resolution. Due to operational or quality issues, many data are missing on certain dates.

Study region. Our study region in southwestern Minnesota covers 490,000 locations at the resolution of Sentinel data, which cover an area of 4,900 ha. We establish the mapping between MODIS and Sentinel and gather the MODIS data for each location. The involved MODIS data in total cover 1,236 MODIS pixels. In the experiment, we only consider major crop types planted in this region, which include corn, soybean and sugarbeet, as their ground-truth labels in USDA crop data layer product are more accurate than other minor crops. Among all the locations in our study region, 217,435 locations are corns, 101,883 locations are soybeans and 96,612 locations are sugarbeets.

\subsection{Classification}

We first evaluate the classification performance of MARSM. In our implementation, we conduct pixel-wise classification for each location/pixel at Sentinel level $(10 \mathrm{~m} \times 10 \mathrm{~m})$. We use two-layer neural networks for functions $\varphi^{\text {gen }}, \varphi^{\text {prior }}$ and $\varphi^{\text {prior }}$ with 120 hidden variables for Sentinel and 80 hidden variables for MODIS. The dimension of the latent variable $z$ is 80 and 60 for Sentinel data and MODIS data, respectively. The hyper-parameter $\lambda$ in adversarial learning is set to 0.2 .

We compare MARSM to multiple baselines, including Artificial Neural Networks (ANN) using concatenated multitemporal data, ensemble single-date Random Forest model (e-RF) [Waske and Braun, 2009], ensemble Fully Convolutional Neural Networks (e-FCNN) [Audebert et al., 2017], and standard LSTM using concatenated multi-scale data at each time step and linear interpolation for missing data.

We also compare against baselines that impute missing data, including GRU-D [Che et al., 2016], and LSTM models using the generated missing data by a temporal-example 
Proceedings of the Twenty-Eighth International Joint Conference on Artificial Intelligence (IJCAI-19)

\begin{tabular}{|c|c|c|c|c|c|c|c|c|c|c|c|c|c|c|c|c|}
\hline \multirow[b]{3}{*}{ Method } & \multicolumn{8}{|c|}{ Entire-region test } & \multicolumn{8}{|c|}{ Cross-region test } \\
\hline & \multicolumn{4}{|c|}{$T A$} & \multicolumn{4}{|c|}{$T B$} & \multicolumn{4}{|c|}{$T A$} & \multicolumn{4}{|c|}{$T B$} \\
\hline & Corn & Soy & Sugar & All & Corn & Soy & Sugar & All & Corn & Soy & Sugar & All & Corn & Soy & Sugar & All \\
\hline ANN & 0.80 & 0.03 & 0.01 & 0.66 & 0.80 & 0.33 & 0.42 & 0.69 & 0.61 & 0.23 & 0.17 & 0.59 & 0.63 & 0.00 & 0.33 & 0.64 \\
\hline e-RF & 0.77 & 0.57 & 0.82 & 0.75 & 0.81 & 0.78 & 0.84 & 0.81 & 0.73 & 0.60 & 0.53 & 0.66 & 0.72 & 0.63 & 0.63 & 0.69 \\
\hline e-FCNN & 0.80 & 0.59 & 0.82 & 0.78 & 0.81 & 0.83 & 0.83 & 0.82 & 0.70 & 0.61 & 0.58 & 0.66 & 0.74 & 0.63 & 0.62 & 0.72 \\
\hline LSTM & 0.74 & 0.65 & 0.75 & 0.72 & 0.87 & 0.83 & 0.94 & 0.88 & 0.79 & 0.62 & 0.57 & 0.70 & 0.82 & 0.61 & 0.61 & 0.74 \\
\hline $\mathrm{LSTM}^{t e l}$ & 0.80 & 0.75 & 0.78 & 0.78 & 0.89 & 0.85 & 0.92 & 0.90 & 0.77 & 0.63 & 0.57 & 0.69 & 0.80 & 0.61 & 0.63 & 0.73 \\
\hline $\mathrm{LSTM}^{\text {gen }}$ & 0.84 & 0.75 & 0.82 & 0.82 & 0.92 & 0.90 & 0.91 & 0.93 & 0.78 & 0.65 & 0.59 & 0.73 & 0.82 & 0.69 & 0.56 & 0.76 \\
\hline cLSTM & 0.88 & 0.80 & 0.79 & 0.85 & 0.91 & 0.88 & 0.94 & 0.92 & 0.77 & 0.62 & 0.66 & 0.73 & 0.83 & 0.62 & 0.58 & 0.76 \\
\hline GRU-D & 0.87 & 0.77 & 0.90 & 0.85 & 0.94 & 0.82 & 0.92 & 0.92 & 0.69 & 0.22 & 0.03 & 0.54 & 0.65 & 0.47 & 0.69 & 0.59 \\
\hline MARSM $^{\text {stn }}$ & 0.78 & 0.79 & 0.77 & 0.77 & 0.81 & 0.78 & 0.83 & 0.79 & 0.76 & 0.62 & 0.49 & 0.67 & 0.78 & 0.63 & 0.59 & 0.72 \\
\hline MARSM $^{\text {wadv }}$ & 0.90 & 0.82 & 0.82 & 0.87 & 0.89 & 0.90 & 0.84 & 0.90 & 0.80 & 0.61 & 0.60 & 0.74 & 0.82 & 0.63 & 0.61 & 0.75 \\
\hline MARSM $^{w s p}$ & 0.89 & 0.87 & 0.86 & 0.90 & 0.91 & 0.96 & 0.94 & 0.95 & 0.84 & 0.64 & 0.60 & 0.77 & 0.83 & 0.68 & 0.64 & 0.80 \\
\hline MARSM & 0.92 & 0.89 & 0.88 & 0.92 & 0.92 & 0.97 & 0.93 & 0.96 & 0.84 & 0.64 & 0.64 & 0.78 & 0.88 & 0.63 & 0.65 & 0.80 \\
\hline
\end{tabular}

Table 1: The entire-region test and the cross-region test for each period $\{T A, T B\}$ using F1-score for each crop and overall accuracy (All).

learning(TEL)-based super-resolution approach [Zhang et al., 2017a] $\left(\mathrm{LSTM}^{t e l}\right.$ ) and by the proposed MARSM method $\left(\mathrm{LSTM}^{\text {gen }}\right.$ ). Also, we compare against an LSTM combining multi-scale data in the final layer (cLSTM) following [Chen and Stow, 2003] (using generated data by MARSM).

We finally compare against three variants of MARSM to show the efficacy of several factors: (1) MOD - incorporation of MODIS data, (2) ADV - adversarial regularizer, and (3) SP - spatial smoothing in classification. These variants are MARSM ${ }^{\text {stn }}$ (no MOD, ADV or SP), MARSM ${ }^{\text {wadv }}$ (with MOD), MARSM ${ }^{w s p}$ (with MOD+ADV).

We test each method on two periods in 2016: TA - Jan 06 to May 25 with Sentinel data missing on Feb 25, Mar 06, and Mar 16, and $T B$ - Apr 05 to Nov 11 with Sentinel data missing on July 04 and July 14 . Note that $T A$ does not cover the crop growing season, but crop residues still show distinctions among different varieties.

For each period, we measure the classification performance by randomly selecting $60 \%$ data for training and then testing on the remaining $40 \%$ data. Also, we evaluate the performance in two scenarios: 1) entire-region test: the training and testing data are sampled from the same region, i.e., the entire study region, and 2) cross-region test: we randomly select a continuous test region, and then take training samples which do not overlap with test region.

According to Table 1, we observe that the performance in cross-region test is generally worse than that in entire-region test since farmers in different places have different preference in planting and harvesting crops. Besides, the performance in $T B$ is generally better than $T A$, as $T B$ covers the growing season, which shows more distinctive characteristics of crops.

In both tests, MARSM outperforms the other baselines. The comparison between MARSM ${ }^{\text {stn }}$ and MARSM ${ }^{\text {wadv }}$ shows that MODIS data can provide promising insights in learning the temporal patterns which contribute to missing data generation and classification. The improvement from MARSM $^{\text {wadv }}$ to MARSM ${ }^{w s p}$ shows the effectiveness of the adversarial regularizer. In addition, MARSM outperforms MARSM $^{w s p}$ because it incorporates the spatial smoothing.

Besides, we can observe that ANN does not perform as well as other sequential models. This is because different crops look similar in many single dates. Moreover, the ensemble single-date methods e-RF and e-FCNN do not perform well because they do not make use of the temporal growing patterns. Also, e-RF does not model any interactions between data from two sources.

The comparison between $\left\{\right.$ LSTM, LSTM $\left.{ }^{\text {tel }}\right\}$ and $\left\{\right.$ LSTM $^{\text {gen }}$, LSTM $\left.^{\text {comb }}\right\}$ demonstrates that MARSM can generate high-quality data. The direct interpolation used in LSTM baseline or the TEL-based super-resolution approach in LSTM $^{\text {tel }}$ suffer from the heavy noise that exists at individual locations in Sentinel data. Also, they can hardly capture the variability of crops. Overall, these methods have lower accuracy than MARSM since the error for generated data can be further accumulated to classification. The method GRU-D imputes missing data based on a decaying factor determined by the data. This method performs well in the entire-region test but cannot generalize to different test regions with shift on the feature space.

\subsection{Generated Missing Data}

We zoom into a small region and show the generated data on Jul 14 (Fig. 3 (a)). Due to the heavy noise in Sentinel data and the complex relationships among multiple spectral bands, the generated data is slightly blurry compared to true data. Nevertheless, the generated data capture the boundaries of croplands and distinctions between different crops, which are critical to the classification.

To quantify the performance of data generation, we evaluate MARSM, MARSM ${ }^{\text {wadv }}$ and MARSM ${ }^{s t n}$ by comparing the generated data with true data. Specifically, for each method, we generate data from Jul 24 to Sep 2 (five time steps). Then we measure the average absolute distance between generated data and true data over all the locations. The results are shown in Fig. 3 (b). We observe that the generated data by MARSM stay close to true data over all the five steps. Without adversarial regularizer, MARSM ${ }^{\text {wadv }}$ does not perform as well as MARSM, especially after more time steps. On the other hand, as MODIS data provide information of temporal evolution and mitigate the noise in Sentinel data, the removal of MODIS data leads to the generation of less accurate data, as can be seen by the performance of MARSM ${ }^{s t n}$. 


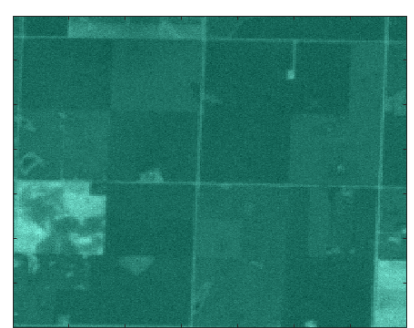

(a)

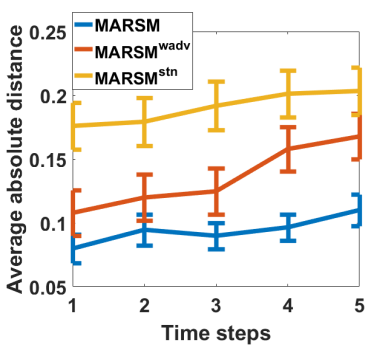

(b)
Figure 3: (a) The generated data on Jul 14 in an example region (shown by a specific spectral band). (b) The average absolute distance between generated data and true data from Jul 24 to Sep 2. The error bar represents the \pm standard deviation.

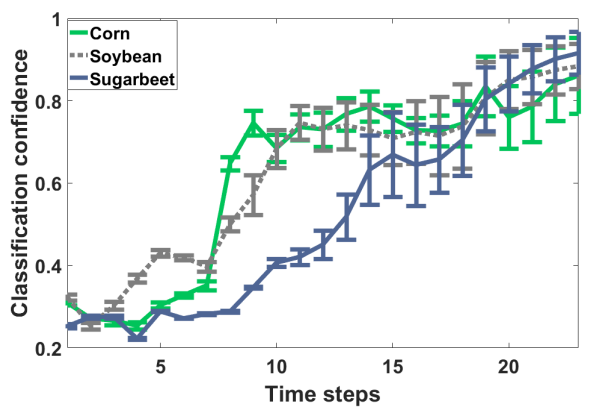

Figure 4: Confidence progression for different crop types over time. The error bar represents the \pm standard deviation.

\subsection{Confidence Progression}

Here we apply MARSM from Apr 05 to Nov 11 (23 time steps). For each class of corn, soybean, and sugarbeet, we measure the classification confidence (averaged over corresponding samples) over time, as shown in Fig. 4.

Our method captures that corn samples quickly gain confidence at the $8^{\text {th }}$ and $9^{\text {th }}$ time steps, which correspond to Jun $14 \sim$ Jun 24 . To validate the correctness of this finding, we show the RGB image of an example region captured on Jun 24 in Fig. 5 (a). We can clearly see that in the early growing season, corn turns into green more quickly than soybean and sugarbeet, and therefore can be identified in this period.

Fig. 4 shows MARSM detects that sugarbeets still gain confidence after October. We show another RGB image on Oct 05 (the same example region) in Fig. 5 (b). While corns and soybeans have been harvested, sugarbeets still remain green. This demonstrates that MARSM can successfully capture the periods with discriminative knowledge and quickly gains confidence in these periods.

\section{Related Work}

Many existing works map croplands by using MODIS data at 250/500m spatial resolution [Inglada et al., 2016; Zhong et al., 2016], but they cannot identify small crop patches that widely exist in the world. Some other works directly apply machine learning algorithms on high-resolution imagery

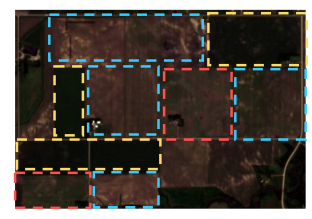

(a)

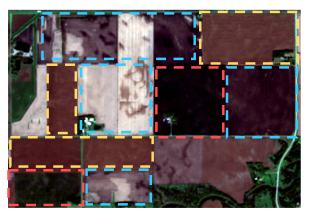

(b)
Figure 5: The satellite imagery in RBG captured on (a) Jun 24 and (b) Oct 05. Color legend for blocks: yellow - corn, blue - soybean, red - sugarbeet.

(e.g., Landsat and Sentinel) [Zhong et al., 2014; Inglada et al., 2016], or on the extracted object-based or shape-based features [Gueguen and Hamid, 2015; Myint et al., 2011]. However, these works do not well address the challenges in high-resolution data, including natural noise factors, irregular temporal frequency and low data quality. To solve these challenges, researchers have sought for combining data in different resolutions for detection [Chen and Stow, 2003; Kurtz et al., 2012; Audebert et al., 2017]. However, these works only focus on combining data at single snapshots.

With recent advances of deep learning, RNN-based models have shown to be effective in many land cover and environmental problems [Lyu et al., 2016; Jia et al., 2017a; Jia et al., 2017b; Jia et al., 2019b; Jia et al., 2019a; Jia et $a l ., 2019 \mathrm{c}]$. However, due to their fully deterministic internal transition structure, they are inappropriate to model the variability in data with complex dependencies. To this end, VRNN is proposed which introduces randomness via latent variables and models the dependencies between latent variables at neighboring time steps [Chung et al., 2015]. Our proposed method is based on the VRNN model but extends it to handle multi-scale multi-temporal data and track the realtime confidence progression.

\section{Conclusion}

In this paper, we propose a framework MARSM that combines multi-scale remote sensing data to identify croplands. The experimental results demonstrate that MARSM greatly improves the detection by learning from multi-scale data. Also, the generated missing data by MARSM stay close to true data over time, and lead to a better classification. In addition, the obtained confidence progression results conform to the growth patterns of crops through visual validation. With the advances in remote sensing technology, the proposed framework can contribute to a large class of land cover problems, which help promote the understanding of global environmental changes.

MARSM can also be applied to other important applications, such as the disease progression modeling where healthcare data are often collected at different time scales with high missing rate [Yang et al., 2018; Zhang et al., 2017b].

\section{Acknowledgements}

This work was funded by the NSF awards 1838159 and 1739191. Access to computing facilities was provided by Minnesota Supercomputing Institute. 


\section{References}

[Audebert et al., 2017] Nicolas Audebert, Bertrand Le Saux, and Sébastien Lefèvre. Joint learning from earth observation and openstreetmap data to get faster better semantic maps. In EARTHVISION 2017 CVPR Workshop, 2017.

[Che et al., 2016] Zhengping Che, Sanjay Purushotham, Kyunghyun Cho, David Sontag, and Yan Liu. Recurrent neural networks for multivariate time series with missing values. arXiv preprint arXiv:1606.01865, 2016.

[Chen and Stow, 2003] DongMei Chen and Douglas Stow. Strategies for integrating information from multiple spatial resolutions into land-use/land-cover classification routines. $P E \& R S, 2003$.

[Chung et al., 2015] Junyoung Chung, Kyle Kastner, Laurent Dinh, Kratarth Goel, Aaron C Courville, and Yoshua Bengio. A recurrent latent variable model for sequential data. In Advances in neural information processing systems, pages 2980-2988, 2015.

[Gueguen and Hamid, 2015] Lionel Gueguen and Raffay Hamid. Large-scale damage detection using satellite imagery. In $C V P R, 2015$.

[Guindin-Garcia et al., 2012] Noemi Guindin-Garcia, Anatoly A Gitelson, Timothy J Arkebauer, John Shanahan, and Albert Weiss. An evaluation of modis 8-and 16-day composite products for monitoring maize green leaf area index. Agricultural and Forest Meteorology, 161:15-25, 2012.

[Habibie et al., 2017] Ikhsanul Habibie, Daniel Holden, Jonathan Schwarz, Joe Yearsley, Taku Komura, Jun Saito, Ikuo Kusajima, Xi Zhao, Myung-Geol Choi, Ruizhen Hu, et al. A recurrent variational autoencoder for human motion synthesis. IEEE CG\&A, 2017.

[Inglada et al., 2016] Jordi Inglada, Arthur Vincent, Marcela Arias, and Claire Marais-Sicre. Improved early crop type identification by joint use of high temporal resolution sar and optical image time series. Remote Sensing, 2016.

[Jia et al., 2017a] Xiaowei Jia, Ankush Khandelwal, Guruprasad Nayak, James Gerber, Kimberly Carlson, Paul West, and Vipin Kumar. Incremental dual-memory lstm in land cover prediction. In Proceedings of the 23rd ACM SIGKDD International Conference on Knowledge Discovery and Data Mining, pages 867-876. ACM, 2017.

[Jia et al., 2017b] Xiaowei Jia, Ankush Khandelwal, Guruprasad Nayak, James Gerber, Kimberly Carlson, Paul West, and Vipin Kumar. Predict land covers with transition modeling and incremental learning. In SIAM International Conference on Data Mining. SIAM, 2017.

[Jia et al., 2019a] Xiaowei Jia, Sheng Li, Ankush Khandelwal, Guruprasad Nayak, Anuj Karpatne, and Vipin $\mathrm{Ku}-$ mar. Spatial context-aware networks for mining temporal discriminative period in land cover detection. In SIAM International Conference on Data mining. SIAM, 2019.

[Jia et al., 2019b] Xiaowei Jia, Guruprasad Nayak, Ankush Khandelwal, Anuj Karpatne, and Vipin Kumar. Classifying heterogeneous sequential data by cyclic domain adap- tation: An application in land cover detection. In SIAM International Conference on Data mining. SIAM, 2019.

[Jia et al., 2019c] Xiaowei Jia, Jared Willard, Anuj Karpatne, Jordan Read, Jacob Zwart, Michael Steinbach, and Vipin Kumar. Physics guided rnns for modeling dynamical systems: A case study in simulating lake temperature profiles. In Proceedings of the 2019 SIAM International Conference on Data Mining, pages 558-566. SIAM, 2019.

[Kingma and Welling, 2013] Diederik P Kingma and Max Welling. Auto-encoding variational bayes. arXiv preprint arXiv:1312.6114, 2013.

[Kurtz et al., 2012] Camille Kurtz, Nicolas Passat, Pierre Gancarski, and Anne Puissant. Extraction of complex patterns from multiresolution remote sensing images: A hierarchical top-down methodology. Pattern Recognition, 45(2):685-706, 2012.

[Lyu et al., 2016] Haobo Lyu, Hui Lu, and Lichao Mou. Learning a transferable change rule from a recurrent neural network for land cover change detection. Remote Sensing, 2016.

[Mathieu et al., 2015] Michael Mathieu, Camille Couprie, and Yann LeCun. Deep multi-scale video prediction beyond mean square error. arXiv preprint:1511.05440, 2015.

[Myint et al., 2011] Soe W Myint, Patricia Gober, Anthony Brazel, Susanne Grossman-Clarke, and Qihao Weng. Perpixel vs. object-based classification of urban land cover extraction using high spatial resolution imagery. Remote sensing of environment, 115(5):1145-1161, 2011.

[Waske and Braun, 2009] Björn Waske and Matthias Braun. Classifier ensembles for land cover mapping using multitemporal sar imagery. ISPRS Journal of Photogrammetry and Remote Sensing, 64(5):450-457, 2009.

[Yang et al., 2018] Xi Yang, Yuan Zhang, and Min Chi. Time-aware subgroup matrix decomposition: Imputing missing data using forecasting events. In 2018 IEEE International Conference on Big Data. IEEE, 2018.

[Zhang et al., 2017a] Yihang Zhang, Peter M Atkinson, Xiaodong Li, Feng Ling, Qunming Wang, and Yun Du. Learning-based spatial-temporal superresolution mapping of forest cover with modis images. IEEE Transactions on Geoscience and Remote Sensing, 55(1):600-614, 2017.

[Zhang et al., 2017b] Yuan Zhang, Chen Lin, Min Chi, Julie Ivy, Muge Capan, and Jeanne M Huddleston. Lstm for septic shock: Adding unreliable labels to reliable predictions. In IEEE Big Data. IEEE, 2017.

[Zhong et al., 2014] Liheng Zhong, Peng Gong, and Gregory $\mathrm{S}$ Biging. Efficient corn and soybean mapping with temporal extendability: A multi-year experiment using landsat imagery. RSE, 2014.

[Zhong et al., 2016] Liheng Zhong, Lina Hu, Le Yu, Peng Gong, and Gregory S Biging. Automated mapping of soybean and corn using phenology. ISPRS Journal of Photogrammetry and Remote Sensing, 2016. 